\title{
FLUORIDE RELEASE AND RECHARGING ABILITY OF GLASS IONOMER CEMENT INCORPORATING HYDROXYAPATITE NANOPARTICLES
}

\author{
Nermin Alsayed Mahmoud* and Asmaa Abdelhakeem Metwally**
}

\begin{abstract}
Aim: The purpose of this study was to evaluate fluoride release and recharging ability of glass ionomer cement incorporating hydroxyapatite nanoparticles.

Materials and methods: Hydroxyapatite nanoparticles (NHA) were prepared by wet chemical method, the experimental material powder (GIC- NHA hybrid) was made by combining different weight percent $(1 \%, 5 \%$ and $10 \%)$ of nano-hydroxyapatite (NHA) powder with GIC powder. Forty disks were prepared and divided into 4 groups, ten specimens from each tested material $(n=10)$, Control group standard GIC, GIC with 1\% NHA, GIC with 5\% NHA and GIC with $10 \%$ NHA. Each specimen was placed in a plastic tube containing $1 \mathrm{ml}$ deionized water, stored at $37^{\circ} \mathrm{C}$ in an incubator and measurements of fluoride release were determined one day, one week, two week and one month using multi-parameter bench photometer. For evaluation of recharge ability, refluoridation of each tested disc was carried on day 30 after measurement of fluoride release and the previous procedures of fluoride release measurements were repeated again.
\end{abstract}

Results: Before and after recharge there was a statistically significant difference in the mean value of fluoride ion release between the control and (1\%) groups, as well as a statistically significant difference between the control group and both (5\% and 10\%) groups, with the highest mean value recorded in $(10 \%)$ group. Between the different testing intervals used in the study, there was a statistically significant difference where $(\mathrm{P}<0.001)$.

Conclusion: incorporation of $10 \%$ nano-hydroxyapatite particles within glass ionomer cement increased fluoride ion release from GIC and its recharging ability.

KEYWORDS: Fluoride release, Fluoride recharge, Glass Ionomer Cement Hydroxyapatite Nanoparticles

\footnotetext{
* Lecturer of Operative Dentistry Department, Faculty of Dentistry, Minia University

** Lecturer of Biomaterial, Faculty of Dentistry, Minia University
} 


\section{INTRODUCTION}

Glass ionomer cement (GIC) is unique among dental materials because its fluoride release, good biocompatibility, and chemical bond to tooth structure with no need for etch and primer. When a tooth-colored material is selected, it can be employed in a variety of applications and because of its fluoride release property, it may help prevent cavities. It's frequently advised for use as a temporary or permanent restoration in caries-prone people. ${ }^{(1)}$

Low mechanical qualities and sensitivity to moisture, on the other hand, have been a key obstacle to this restorative material's broad clinical use. ${ }^{(2)} \mathrm{A}$ variety of modifications have been made to GIC powder to improve the mechanical qualities of the restorative material, including the addition of additives (metal, glass and different nonreactive particles) as fillers in GIC matrix. These initial modifications increased the mechanical properties without having a negative effect on fluoride release ability of the material. ${ }^{(3)}$

Despite GICs' good biocompatibility, several attempts have been made to improve their bioactivity by adding biologically active glasses. Hydroxyapatite (HA) possesses excellent biological characteristics, with a composition and crystal structure that is similar to natural apatite found in human dental hard tissues and the skeletal system. ${ }^{(4)}$

Nano-HA crystals have been found to promote enamel remineralization in recent research. The increased mechanical properties of nano-HA-added GIC have also been attributed to nano-HA. The ionic interaction between the polyacrylic acid and the apatite crystals was thought to be responsible for the enhancement in mechanical characteristics of nano-HA-added GIC. ${ }^{(5,6)}$

As a result, the goal of this research was to assess the effect of hydroxyapatite nanoparticles incorporation on fluoride release and recharging of GIC.

\section{MATERIALS \& METHODS}

\section{Preparation of hydroxyapatite nanoparticles:}

According to (Irfan etal.,2020)(7) ${ }^{(7)}$, hydroxyapatite nanoparticles were made by reacting calcium nitrate-tetrahydrate $(\mathrm{Ca}(\mathrm{NO} 3) 2.4 \mathrm{H} 2 \mathrm{O})$ with diammonium hydrogen phosphate ((NH4)2HPO4) using a wet chemical technique. The (NH4)2HPO4 aqueous solution was added to the $\mathrm{Ca}(\mathrm{NO} 3) 2.4 \mathrm{H} 2 \mathrm{O}$ aqueous solution at room temperature, with $\mathrm{pH}$ values ranging between 10 and 12 . The precipitates were placed in an autoclave and hydrothermally treated for 2 hours at $0.3 \mathrm{MPa}$ pressure and $140^{\circ} \mathrm{C}$.

The average size and form of the produced nanoparticles materials were determined using a transmission electron microscope (TEM) (JEOL JEM-2100, Tokyo, Japan). Furthermore Ultraviolet visible near infrared Fiber optics spectrophotometer (Ocean Optics USB2000+VIS-NIR, Florida, USA) has been used to monitor the absorption rate of the used nanoparticles material. TEM showed needle shaped nanoparticles with $150 \mathrm{~nm} \pm 30 \mathrm{~nm}$ in size fig. (1).

\section{Preparation of GIC- NHA hybrid}

Experimental material powder was prepared via mixing different weight percent (1,5 and 10) of nano-hydroxyapatite (NHA) powder with GIC

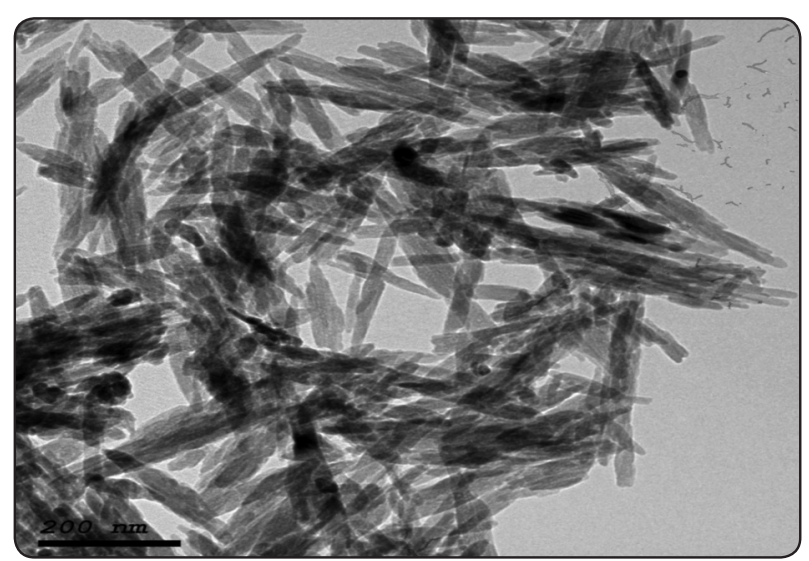

Fig. (1) TEM image of needle shaped hydroxyapatite nanoparticles 
powder (GC Gold Label 9 Posterior Restorative, GC Corporation Tokyo, Jaban) .

The fNHA powder was mixed with GIC powder by plastic spatula on a glass slab and for even distribution of nano hydroxyapatite particles through the glass ionomer powder, the mixed powder was remixed in plastic capsules by amalgamator (IMIXM3, Zhenhal, China) for 10s. The powder containing (1, 5 and 10) wt\% NHA and GIC material liquid were mixed according to manufacturer's instructions $[\mathrm{P} / \mathrm{L}$ ratio $(3.6 \mathrm{~g} / 1.0 \mathrm{~g})]$.

\section{Preparation and grouping of the specimens}

Forty disks were prepared and divided into 4 groups, ten specimen from each tested material $(\mathrm{n}=10)$, Control group standard GIC (Group I), GIC with $1 \%$ nano hydroxyapatite (Group II), GIC with 5\% nano hydroxyapatite (Group III) and GIC with $10 \%$ nano hydroxyapatite (Group IV).

Each specimen was prepared by mixing the GICNHA hybrid material according to manufacturer's instructions, then placed in a split Teflon mold with a central hole having dimensions of $(10 \mathrm{~mm}$ diameter and $2 \mathrm{~mm}$ height) and compressed by glass slab until setting occur then excess material was removed after removal from the mold.

\section{Fluoride release test}

Each specimen was placed in a plastic tube containing $1 \mathrm{ml}$ deionized water and stored in an incubator (PS. 3A, Advanced Technology, Egypt) at $37^{\circ} \mathrm{C}$, the fluoride release measurements were determined after one day, one week, two weeks and one month.

Concentration of fluoride in each sample solution was measured using Multi-parameter bench photometer (HI83200, HANNA Instruments, USA) by selecting the fluoride method according to manufacture instructions. Two cuvettes were filled with $2 \mathrm{~mL}$ of HI 93729-0 SPADNS Reagent and one cuvette with distilled water. To mix, the cap was replaced and inverted multiple times. The sample was placed in the other cuvette. To mix, the cap was replaced and inverted multiple times. The cuvette was placed in the holder with the reacted distilled water (\# 1) and the lid was closed.

There was a timer and a display. Alternatively, two minutes were waited for and then pushed Zero to show the countdown before zeroing the blank. When the meter was zeroed and ready for use, the display displayed "-0.0-." Remove the cuvette from the mix. The reacted sample was placed in the other cuvette (\# 2), which was introduced into the instrument. The button to begin reading was pressed. The data are displayed in milligrams per liter of fluoride.

\section{Fluoride recharging ability Test}

Re-fluoridation of each tested disc was carried as follow, on day 30 after measurement of fluoride release. The discs of each tested group were cleansed by rinsing them three times with $5 \mathrm{ml}$ deionized water, dried for $5 \mathrm{~min}$ by filter paper, then immersed in $0.2 \% \mathrm{NaF}$ solution of $10 \mathrm{ml}$ for 5 minutes (Dimnysopoulos et al., 2013). ${ }^{(8)} 0.2 \% \mathrm{NaF}$ solution prepared by dissolving $2 \mathrm{gm}$ of $\mathrm{NaF}$ powder in $1 \mathrm{~L}$ of distilled water in a plastic bottle.

The discs were rinsed with $5 \mathrm{ml}$ deionized water and then each disc was placed in a glass tube containing $1 \mathrm{ml}$ deionized water and stored in an incubator at $37^{\circ} \mathrm{C}$. The previous procedures of fluoride release were repeated again. The fluoride release measurements were determined after one day, one week, two weeks, and one month. Fluoride concentration in each sample solution was measured using multiparameter bench photometer.

\section{Statistical analysis}

In each test, the mean and standard deviation values were computed for each group. Using the Kolmogorov Smirnov and Shapiro-Wilk tests, the data were shown to have a parametric (normal) distribution. 
To compare more than two groups in unrelated samples, a two-way ANOVA was performed, followed by a Tukey post hoc test. To compare two groups in unrelated samples, an independent sample t-test was performed. To compare more than two groups in linked samples, the repeated measure ANOVA test was performed. To compare two groups in related samples, a paired sample t-test was performed.

$\mathrm{P} \leq 0.05$ was used as the significant level. IBM ${ }^{\circledR}$ SPSS ${ }^{\circledR}$ Statistics Version 20 for Windows was used to conduct the statistical analysis.

\section{RESULTS}

\section{Fluoride release before recharging}

Table (1) and fig. (2) indicate the mean and SD values of fluoride release before recharging of the studied materials at various test durations.

After one day, there was no statistically significant difference between (Control) and $(1 \%)(\mathrm{p}=0.157)$, however there was a statistically significant difference between (Control) and each of the (5\%) and (10\%) groups (p0.001). After one week, there was a statistically significant difference between (Control) and each of the (1\%), (5\%) and $(10 \%)$ groups $(\mathrm{p}<0.001)$; however, there was no statistically significant difference between (5\%) and $(10 \%)$ groups $(\mathrm{p}=0.493)$. After two weeks, there was no statistically significant difference between (Control) and (1\%) ( $\mathrm{p}=0.998)$, but there was a statistically significant difference between (Control) and each of the (5\%) and (10\%) groups (p0.001). After one month, no statistically significant difference was found between (Control) and (5\%) groups ( $\mathrm{p}=0.996$ ), however a statistically significant difference was identified between (Control) and each of the $(1 \%)$ and $(10 \%)$ groups $(\mathrm{p}<0.001)$. There was a statistically significant difference in fluoride release between the groups at different testing intervals (one day, one week, two weeks and one month) with the highest mean value reported after one day $(\mathrm{p}<0.001)$ followed by gradual decrease along the testing period.

\section{Fluoride release after recharging}

Table (2) and fig. (3) indicate the mean and SD values of fluoride release before recharging of the studied materials at various test durations.

TABLE (1): Mean and standard deviation (SD) values of fluoride release before recharging of various groups:

\begin{tabular}{|c|c|c|c|c|c|c|c|c|c|}
\hline \multirow{3}{*}{ Variables } & \multicolumn{9}{|c|}{ Fluoride release before recharging } \\
\hline & \multicolumn{2}{|c|}{ Control } & \multicolumn{2}{|c|}{$1 \%$} & \multicolumn{2}{|c|}{$5 \%$} & \multicolumn{2}{|c|}{$10 \%$} & \multirow{2}{*}{ p-value } \\
\hline & Mean & SD & Mean & SD & Mean & SD & Mean & SD & \\
\hline 1 day & $24.52^{\mathrm{aC}}$ & 0.69 & $21.28^{\mathrm{aC}}$ & 1.53 & $47.64^{\mathrm{aB}}$ & 1.55 & $54.48^{\mathrm{aA}}$ & 3.99 & $<0.001 *$ \\
\hline 1 week & $13.22^{\mathrm{bC}}$ & 1.95 & $18.44^{\mathrm{bB}}$ & 1.51 & $22.04^{\mathrm{cA}}$ & 1.41 & $23.68^{\mathrm{cA}}$ & 2.21 & $<0.001 *$ \\
\hline 2 weeks & $5.82^{\mathrm{cB}}$ & 0.90 & $5.92^{\mathrm{cB}}$ & 0.82 & $12.16^{\mathrm{dA}}$ & 2.44 & $14.14^{\mathrm{dA}}$ & 2.62 & $<0.001 *$ \\
\hline 1 month & $5.48^{\mathrm{cB}}$ & 1.30 & $3.54^{\mathrm{dC}}$ & 0.74 & $5.62^{\mathrm{eB}}$ & 0.64 & $8.36^{\mathrm{eA}}$ & 1.19 & $<0.001 *$ \\
\hline p-value & \multicolumn{2}{|c|}{$<0.001 *$} & \multicolumn{2}{|c|}{$<0.001 *$} & \multicolumn{2}{|c|}{$<0.001 *$} & \multicolumn{2}{|c|}{$<0.001 *$} & \\
\hline
\end{tabular}

Significant difference is shown by means with different small letters in the same column; significant difference is indicated by means with different capital letters in the same row.

* significant $(p<0.05)$ ns; non-significant $(p>0.05)$ 
TABLE (2): Mean and standard deviation (SD) values of fluoride release after recharging of various groups:

\begin{tabular}{|c|c|c|c|c|c|c|c|c|c|}
\hline \multirow{3}{*}{ Variables } & \multicolumn{9}{|c|}{ Fluoride release after recharging } \\
\hline & \multicolumn{2}{|c|}{ Control } & \multicolumn{2}{|c|}{$1 \%$} & \multicolumn{2}{|c|}{$5 \%$} & \multicolumn{2}{|c|}{$10 \%$} & \multirow{2}{*}{ p-value } \\
\hline & Mean & SD & Mean & SD & Mean & SD & Mean & SD & \\
\hline 1 day & $13.86^{\mathrm{Ca}}$ & 0.27 & $15.93^{\mathrm{Ca}}$ & 0.21 & $18.14^{\mathrm{Ba}}$ & 0.28 & $24.01^{\mathrm{Aa}}$ & 0.49 & $<0.001 *$ \\
\hline 1 week & $10.56^{\mathrm{Cb}}$ & 0.35 & $11.66^{\mathrm{Cb}}$ & 0.28 & $13.48^{\mathrm{Bb}}$ & 0.26 & $17.77^{\mathrm{Ab}}$ & 0.27 & $<0.001 *$ \\
\hline 2 weeks & $6.79^{\mathrm{Bc}}$ & 0.40 & $8.07^{\mathrm{Bc}}$ & 0.15 & $9.15^{\mathrm{Bc}}$ & 0.20 & $12.82^{\mathrm{Ac}}$ & 0.26 & $<0.001 *$ \\
\hline 1 month & $3.65^{\mathrm{Cd}}$ & 0.31 & $6.04^{\mathrm{Bc}}$ & 0.57 & $6.08^{\mathrm{Bd}}$ & 0.17 & $7.16^{\mathrm{Ad}}$ & 0.31 & $<0.001 *$ \\
\hline p-value & \multicolumn{2}{|c|}{$<0.001 *$} & \multicolumn{2}{|c|}{$<0.001 *$} & \multicolumn{2}{|c|}{$<0.001 *$} & \multicolumn{2}{|c|}{$<0.001 *$} & \\
\hline
\end{tabular}

Significant difference is shown by means with different small letters in the same column; significant difference is indicated by means with different capital letters in the same row.

* significant $(p<0.05) n s ;$ non-significant $(p>0.05)$

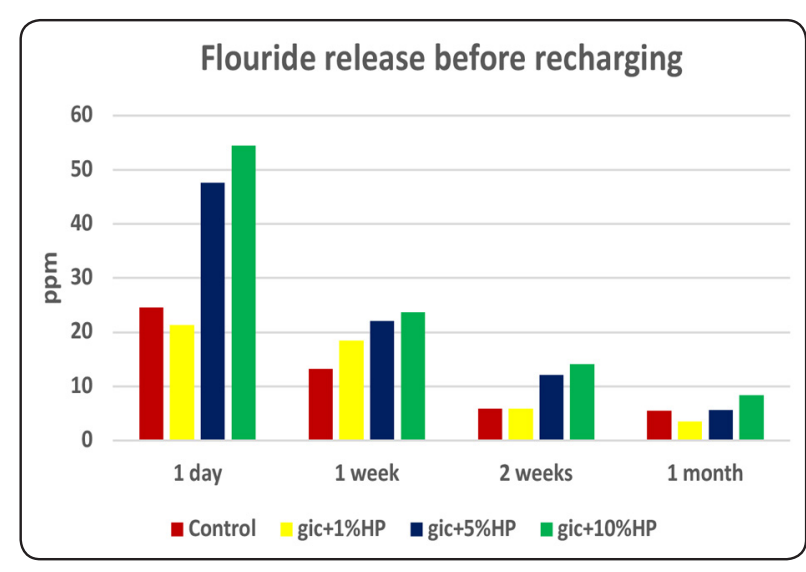

Fig. (2): Bar chart representing fluoride releas e before recharging of different groups.

After one day and after one week of fluoride recharge, no statistically significant difference was found between (Control) and (1\%) ( $=0.298$ \& $\mathrm{p}=0.208$ ) respectively but there was a statistically significant difference between (Control) and each of the $(5 \%)$ and $(10 \%)$ groups $(\mathrm{p}<0.001)$. After two weeks, no statistically significant difference was found between (Control), (1\%) and (5\%) ( $\mathrm{p}=0.377$, $\mathrm{p}=0.695 \& \mathrm{p}=0.267)$ respectively. However a statistically significant difference was found between $(10 \%)$ group and the other groups $(\mathrm{p}<0.001)$. After

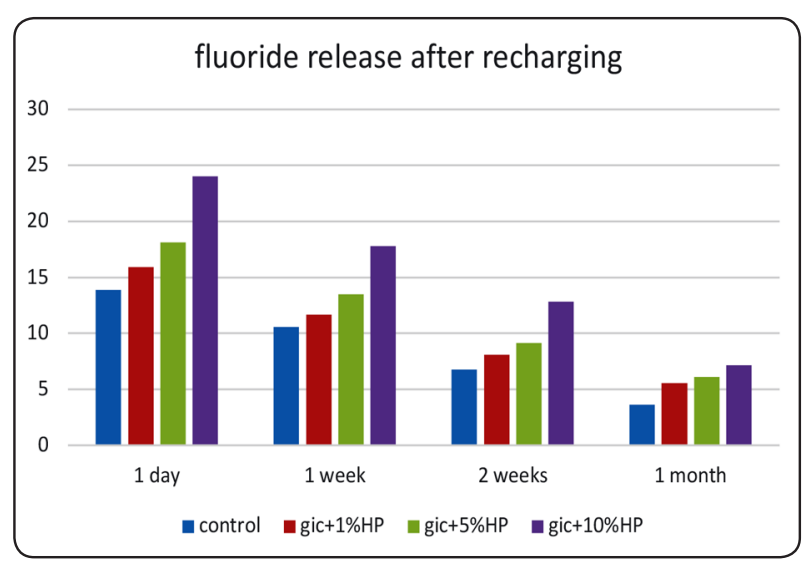

Fig. (3): Bar chart representing fluoride release after recharging of different groups.

one month results showed a statistically significant difference was found between (Control) and $(1 \%),(5 \%)$ and $(10 \%)$ groups $(\mathrm{p}<0.001)$ while no statistically significant difference found between $(1 \%)$ and $(5 \%)$ groups $(\mathrm{P}=0.995)$. A statistically significant difference was found between fluoride release of the different groups after recharge at the different testing periods (one day, one week, two weeks and one month $(p<0.001)$, the highest mean value recorded after one day followed by gradual decrease along the testing period. 


\section{DISCUSSION}

The purpose of this study was to evaluate fluoride release and recharging ability of glass ionomer cement incorporating hydroxyapatite nanoparticles. Nano-hydroxyapatite powder which used in this study were prepared by the wet chemical method according to Chandrasekar., et al $2013^{(9)} \&$ Esmaeilkhanian et al., 2019. ${ }^{(10)}$ Selection of the wet chemical method to synthesis nanoparticles used in this study because of its relatively huge amount of nano-hydroxyapatite could be prepared, acceptable cost and it is the most available method in Egypt. Selection of needle shaped NHP particles because it resemble the morphology of the original apatite crystals in the dental tissue (Alobeedallaha et al., $2011)^{(11)}$ and selection of the powder form of nanohydroxyapatite to facilitate handling and mixing with glass ionomer powder.

Conventional type of glass ionomer cement (GC Gold Label 9Posterior Restorative) was selected due to its easy handling properties, chemical bonding to tooth structure as well as high releasing of fluoride ion which cause a remineralization effect on tooth structure. The experimental material powder was made by mixing various weight percent $(1,5$, and $10 \%$ ) of nano-hydroxyapatite (HA) powder with GIC powder on a glass slab with a spatula then remixed in plastic capsules by a high-speed amalgamator for $10 \mathrm{~s}$ according to Alatawi et al., $2018 .^{(12)}$

Ten disks of $10 \mathrm{~mm}$ in diameter and $2 \mathrm{~mm}$ thickness from each tested material $(n=10)$ were prepared in a split Teflon mold for fluoride release measurement according to Arita., et al $2011^{(13)}$ and then were immersed in individually numbered tubes, each tube contains $1 \mathrm{ml}$ of deionized water. The tubes were placed in the incubator at $37^{\circ} \mathrm{c}$.

In this study deionized water has been used as a storage media because it reflects well the property of fluoride release of the GIC without any influence of minerals or organic molecules, which might be presented in other storage media. This in consistent with the work of Abdul Quader et al., 2012 ${ }^{(14)}$ and Elgamily et al., 2018. ${ }^{(15)}$

For fluoride release measurement before recharge, The storage water from which the discs had been removed was then analyzed to determine the amount of fluoride release. Two $\mathrm{ml}$ of Fluoride reagent was added to each sample's solution of each disc. The fluoride reagent provided constant background ionic strength, adjusted $\mathrm{PH}$ and gave red tint when reaction between fluoride ions in solution and reagent occurred (Mousavinaasab and Meyers, 2009)! (16)

The fluoride release measurement was carried out by using multiparameter bench photometer (HI 83200, Woonsocket, USA); It is a sort of photometric chemical analysis based on the possibility of developing an absorbing compound from a specific chemical reaction between the sample and the reagents. Because a compound's absorption is only dependent on the wavelength of the incident light beam, a narrow spectral bandwidth as well as a good central wavelength should be chosen to maximize observations. The HI 83200's optical system uses customized subminiature tungsten lamps and narrow-band interference filters to provide exceptional performance and repeatability. A wide range of tests are provided by five measurement channels.

In the present study, the fluoride release test data were determined after one day, one week, two week and one month, collected and statistically analyzed. The results showed the effect of time that; a statistically significant difference was found between (one day), (one week), (two weeks) and (one month) groups $(p<0.001)$. The maximum fluoride release for tested glass-ionomer cements was observed in the first day and then decreased gradually until the end of the test periods for all groups.

Farag, $2015^{(17)}$ Reported that responsible cause of the high level of fluoride release in the first day can mostly due to initial superficial rinsing effect and dissolution of glass particles in poly-alkenoate acid during the setting reaction. Meanwhile, the 
fluoride's capacity to spread via cement pores results in constant fluoride release over the next few days.

Results also showed that addition of nanohydroxyapatite to GIC significantly increased the fluoride release ability. There was a statistically significant difference between (Control), $\mathrm{GIC}+1 \% \mathrm{NH}, \quad \mathrm{GIC}+5 \% \mathrm{NH}$ and $\mathrm{GIC}+10 \% \mathrm{NH}$ groups where $(p<0.001)$. The highest mean value of fluoride release was observed in $(\mathrm{GIC}+10 \% \mathrm{NH})$ along the testing period.

Results of this study were consistent with results of Arita., et al 2011 ${ }^{(13)}$ and Chiu et al., 2017 ${ }^{(18)}$ who supposed that the responsible cause of higher values of fluoride release from HA-modified GICs was due to the HA particles which play an important role in increasing the fluoride release based on a reaction between HA particles and the matrix of GIC or glass core. Because of their porosity, they believed that HA particles acted as a channel for fluoride ion release. Also the results were in agreement with the that of Alatawi et al., $2018^{(12)}$ who supposed that the responsible cause of higher values of fluoride release from HA-modified GICs was due to the NHA particles had a significant surface area due to their small size and its addition to GIC increased the acid-base reaction activity, resulting in increased fluoride ion release.

GIC and their derivatives not only release fluoride but also act as a fluoride reservoir. Recharging was described as surface phenomenon, where the adsorbed fluoride ions to the surface of the restoration get washed off. Recharging agents include; fluoridated dentifrices, mouth rinses, fluoride gels and fluoride varnishes. Fluoride mouth rinse remains the most widely recommended routine home care oral hygiene due to its anticariogenic properties and ease of use. Fluoride mouth rinses are based on neutral sodium fluoride solutions in the range from 0.05 to $0.2 \%$ (225-1,000 ppm). Diaz et al., $1995^{(19)}$ and Vermeersch et al., 2001. ${ }^{(20)}$

In this study refluoridation of each tested disc was carried as follow, on day 30 after measurement of fluoride release. The discs of each tested group were cleansed by rinsing them three times with $5 \mathrm{ml}$ deionized water, dried for $5 \mathrm{~min}$ by filter paper, then immersed in $10 \mathrm{ml}$ of $0.2 \% \mathrm{NaF}$ solution which contain about $1000 \mathrm{ppm}$ of fluoride for 5 minute to stimulate the fluoride ion recharge and it was the most effective fluoride agent at recharging according to Han et al., 2002 ${ }^{(21)}$; Dimnysopoulos et al., 2003(8); and Chole et al., 2015.(22)

Fluoride release measurements after recharge were determined after one day, one week, two weeks and one month. The concentration of fluoride in each sample solution was measured using Multiparameter bench photometer.

Results of this study showed that a statistically significant difference was found between control group and both of $(5 \%)$ and $(10 \%)(p<0.001$ with highest mean value recorded for $(10 \%)$ group all over the testing period. none statistical significant difference was found between control group and (1\%) group after one day, one week and two weeks $(\mathrm{p}=0.298 \& \mathrm{p}=0.208$ and $\mathrm{p}=0.695)$ respectively and a statistically significant difference was found between fluoride release of the different groups after recharge at the different testing periods (one day, one week, two weeks and one month $(p<0.001)$, the highest mean value recorded after one day followed by gradual decrease along the testing period.

These results were similar to that found by Arita et al., $2011^{(13)}$, reported that incorporation of $8 \%$ NHP into GIC enhance the chemical characteristics of the material (fluoride release and recharge), and differ than that conducted by Panighari et al., 2016(23) , they found that Hydroxyapatite incorporated GIC As with traditional GIC, it was discovered to be capable of fluoride uptake and subsequent release. Following recharge, both HAGIC and conventional GIC showed an initial burst of fluoride concentration, which continued to rise until the third day. After that, it began to decline until the seventh day, when it eventually reached a plateau that lasted until the twenty-first day. 
Results of all the study groups showed an increased amount of fluoride re-release after 1 day, The fluoride release decrease rapidly suggesting that fluoride release after topical fluoride application represents a washout of ions adsorbed to the surface, rather than an actual diffusion into the matrix as. These results in agreement with that found by Nagi et al., $2018^{(24)}$, who explained that, the brief fluoride recharge period (4 minutes) that was applied once to the specimens in their investigation was responsible for that the only surface parts of the specimens were recharged in their investigation.

The ability of glass ionomer cements to recharge has been hypothesized to be dependent on the glass component, specifically the structure of the hydrogel layer around glass filler particles, which is generated by interactions between fluoridated glass particles and polyacrylic acids ${ }^{(25)}$. Results after recharge were similar to that before recharge as $\mathrm{GIC}+10 \%$ NHP revealed the highest mean value of fluoride ion release than other groups, this is explained by Mousavinasab and Meyers, $2009^{(16)}$ they suggested that high fluoride release material has a higher fluoride recharging ability .

\section{CONCLUSION}

Within the limitations of this study, it could be concluded that incorporation of $10 \%$ nano-hydroxyapatite particles within glass ionomer cement increased fluoride ion release from GIC and its recharging ability which enhance the remineralization effect and anti-cariogenic potential of GIC.

\section{RECOMMENDATION}

1- Enhancement of fluoride ion release and recharging ability of GIC after incorporation of 10 $\%$ NHP recommend its use as a caries controlling restoration.

2- Further investigations are required to evaluate the effect of Incorporation of nano-hydroxyapatite particles within glass ionomer cement on color stability, surface roughness and antibacterial activity etc.

\section{REFERENCES}

1- Ching HS, Luddin N, Kannan TP, Ab Rahman I, Abdul Ghani NR. Modification of glass ionomer cements on their physical-mechanical and antimicrobial properties. J Esthet Restor Dent 2018;30(6):557-571.

2- Berg JH, Croll TP. Glass ionomer restorative cement systems: an update. Pediatr Dent 2015;37(2):116-124.

3- Elsaka SE, Hamouda IM, Swain MV. Titanium dioxide nanoparticles addition to a conventional glass-ionomer restorative: influence on physical and antibacterial properties. J Dent 2011;39(9):589-598.

4- Farooq I, Moheet IA, AlShwaimi E. In vitro dentin tubule occlusion and remineralization competence of various toothpastes. Arch Oral Biol 2015;60(9):1246-1253.

5- Huang S, Gao S, Cheng L, Yu H. Remineralization potential of nano-hydroxyapatite on initial enamel lesions: an in vitro study. Caries Res 2011;45(5):460-468.

6- Moshaverinia A, Ansari S, Moshaverinia M, Roohpour N, Darr JA, Rehman I. Effects of incorporation of hydroxyapatite and fluoroapatite nanobioceramics into conventional glass ionomer cements (GIC) Acta Biomater 2008;4(2):432-440.

7- Irfan Md., Suprajaa P. S., Baraneedharan P., Reddy B.M. A Comparative Study of Nanohydroxyapetite Obtained from Natural Shells and Wet Chemical Process. Journal of Materials Science \& Surface Engineering 2020, 7(1): 938-943.

8- Dionysopoulos D, Koliniotou-Koumpia E, HelvatzoglouAntoniades M, Kotsanos N."Fluoride release and recharge abilities of contemporary fluoride-containing restorative materials and dental adhesives". Dent Mater Journal 2013; 32:296-304.

9- Chandrasekar A., Suresh S. and Arivuoli D. Synthesis and characterization of nano-hydroxyapatite (n-HAP) using the wet chemical technique. International Journal of Physical Sciences.2013; 8(32): 1639-1645.

10- Esmaeilkhanian A., Fariborz S., Aliasghar A.i, Amirreza R., Nader P. Mohammad I. Synthesis and Characterization of Natural Nano-hydroxyapatite Derived from Turkey FemurBone Waste. Applied Biochemistry and Biotechnology. 2019,189:919-932.

11- Alobeedallaha H, Jeffrey L. Ellis b, Ramin R, Hans C and Fariba D. Preparation of Nanostructured Hydroxyapatite 
in Organic Solvents for Clinical Applications. Trends Biomater. Artif. Organs. 2011; 25(1): 12-19.

12- Alatawi R., Elsayed N., Mohamed W. Influence of hydroxyapatite nanoparticles on the properties of glass ionomer cement. Journal of Materials Research \& Technology. 2018;8(1);344-349.

13- Arita K, Yamamoto A, Shinonaga Y, Harada K, Abe Y, Nakagawa et al. Hydroxyapatite particle characteristics influence the enhancement of the mechanical and chemical properties of conventional restorative glassionomer cement. Dental Materials Journal. 2011; 30(6):72-83.

14- Abdul Quader M., Shamsul Alam M., Bashar A. , K. Abdul Gafur, Al-Mansur M. Compressive Strength, Fluoride Release and Recharge of Giomer. Update Dental College journal.2012; 2(2):28-37.

15- Elgamily H., Ghallab O., El-Sayed H. and Nasr M. Antibacterial potency and fluoride release of a glass ionomer restorative material containing different concentrations of natural and chemical products: An in-vitro comparative study. Journal of clinical and experimental dentistry. 2018; 10(4):312-20.

16- Mousavinasab S., Meyers I. "Fluoride release and uptake by glass ionomer cements, compomer and giomer". Res J Biol Sci. 2009; 4:609-16.

17- Farag A. Fluoride release, bond strength and solubility of novel glass ionomer containing chlorhexidine. Egyptian dental journal. 2015.61(4);1-13.

18- Chiu S., Shinonaga Y., Abe Y., Harada K.and Arita K.
Influence of Porous Spherical-Shaped Hydroxyapatite on Mechanical Strength and Bioactive Function of Conventional Glass Ionomer Cement. Materials 2017; 10(27): 1-13.

19- Diaz AM, Holmes DC, Wistrom DW, Swift EJ, Jr. Shortterm fluoride release/uptake of glass ionomer restoratives. Dent Mater 1995;11(2):96-101.

20- Vermeersch G, Leloup G, Vreven J. Fluoride release from glass-ionomer cements, compomers and resin composites. J Oral Rehabil 2001;28(1):26-32.

21- Han L, Edward C, Okamoto A. "A comparative study of fluoride -releasing adhesive resin materials" .Dent Mater J.2002;21:9-19.

22- Chole D.d, Lokhand P., ShashanK k, Bakle S., Amarnath Devagirkar, Praveen Dhore "Comparative Evaluation of the Fluoride Release and Recharge through Four Different Types of Pit and Fissure Sealants: An In Vitro Study”. International Journal of Advanced Health Sciences. 2015; 2: 6.

23- Panigrahi A,. Sudeep S., Sharma S., and Mohanty S. Comparative Evaluation of Fluoride Recharge Ability of Conventional and Hydroxyapatite Modified Glass Ionomer Cement with Daily Low Fluoride Exposure- An Invitro Study. J Clin Diagn Res. 2016; 10(2): 53:55.

24- Nagis., Moharama L., El Hoshyb A. Fluoride release and recharge of enhanced resin modified glass ionomer at different time intervals . Future Dental Journal 2018 (4): 221-224.

25- De Moor RJ, Verbeeck RM, De Maeyer EA. Fluoride release profiles of restorative glass ionomer formulations. Dent Mater. 1996 Mar;12(2):88-95. 